\title{
QUADRATIC JORDAN ALGEBRAS AND CUBING OPERATIONS
}

\author{
BY \\ KEVIN McCRIMMON
}

\begin{abstract}
In this paper we show how the Jordan structure can be derived from the squaring and cubing operations in a quadratic Jordan algebra, and give an alternate axiomatization of unital quadratic Jordan algebras in terms of operator identities involving only a single variable. Using this we define nonunital quadratic Jordan algebras and show they can be imbedded in unital algebras. We show that a noncommutative Jordan algebra $\mathfrak{A}$ (over an arbitrary ring of scalars) determines a quadratic Jordan algebra $\mathfrak{A}^{+}$.
\end{abstract}

In this paper we investigate several connections between quadratic Jordan algebras and cubing operations. We show how the Jordan structure can be derived from the squaring and cubing operations, and that linear maps preserving these operations necessarily preserve the Jordan structure. We show that quadratic maps preserving squares and cubes preserve the Jordan structure too, though this requires some effort. We give three applications of this result. First, we give an alternate axiomatization of a unital quadratic Jordan algebra in terms of operator identities involving only a single variable. Second, using this simpler axiomatization we define quadratic Jordan algebras without units and prove that any such algebra can be imbedded in a unital one. Third, we again use the alternate axiomatization to show that any noncommutative Jordan algebra $\mathfrak{U}$ (over an arbitrary ring of scalars) induces in a natural way a quadratic Jordan algebra $\mathfrak{A}^{+}$.

1. Quadratic algebras. Throughout we adopt the notation of [3]. We fix once and for all a ring of scalars $\Phi$, which can be any commutative associative ring with unit, and consider various algebra structures on a unital $\Phi$-module $X$. A quadratic algebra is a triple $\mathfrak{A}=(X, U, 2)$ where $x \rightarrow x^{2}$ and $x \rightarrow U(x)=U_{x}$ are quadratic mappings of $X$ into $X$ and $\operatorname{Hom}_{\Phi}(X, X)$ respectively. From these we obtain a commutative bilinear product

$$
x \circ y=(x+y)^{2}-x^{2}-y^{2}
$$

and a trilinear product

$$
\left\{\begin{array}{lll}
x & y & z
\end{array}\right\}=U_{x, z} y=\left\{U_{x+z}-U_{x}-U_{z}\right\} y .
$$

Received by the editors July 22, 1969.

AMS 1969 subject classifications. Primary 1740.

Key words and phrases. Jordan algebra, noncommutative Jordan algebra, quadratic Jordan algebra. 
We define auxiliary operators $V_{x}, V_{x, y}$ by

$$
V_{x} y=x \circ y, \quad V_{x, y} z=\{x y z\} .
$$

A homomorphism of quadratic algebras is a linear map $\varphi: \mathfrak{A} \rightarrow \tilde{\mathfrak{A}}$ preserving the two compositions

$$
\varphi\left(x^{2}\right)=\varphi(x)^{\tilde{2}}, \quad \varphi\left(U_{x} y\right)=\widetilde{U}_{\varphi(x)} \varphi(y) .
$$

If $\Omega$ is an extension of $\Phi$ the quadratic $\Phi$-algebra has a natural extension to a quadratic $\Omega$-algebra

$$
\mathfrak{A}_{\Omega}=\Omega \otimes_{\Phi} \mathfrak{A} \text {. }
$$

Any homomorphism $\varphi: \mathfrak{A} \rightarrow \tilde{\mathfrak{A}}$ has a natural extension $\varphi_{\Omega}=1 \otimes \varphi$ to a homomorphism $\varphi_{\Omega}: \mathfrak{A}_{\Omega} \rightarrow \widetilde{\mathfrak{A}}_{\Omega}$. In this way we obtain an extension functor from the category of quadratic $\Phi$-algebras to the category of quadratic $\Omega$-algebras.

A quadratic algebra has a unit $c$ (necessarily unique) if

(1.1) $U_{c}=1$,

(1.2) $V_{c}=2 I$,

(1.3) $U_{x} c=x^{2}$.

Equivalently, we could define a unital quadratic algebra to be a triple $\mathfrak{A}=(X, U, c)$ where $c$ is an element satisfying

(1.1)' $U_{c}=I$,

$(1.2)^{\prime} \quad V_{c, c}=2 I$.

Then we introduce a square by $x^{2}=U_{x} c$, obtaining a quadratic algebra with unit in the sense of (1.1)-(1.3). Conversely, a quadratic algebra with unit satisfies $(1.1)^{\prime}-(1.2)^{\prime}$ since $V_{c, c} x=\left\{\begin{array}{ccc}c & x\}\end{array}=U_{x, c} c=x \circ c\right.$ (by (1.3)) $=V_{c} x$. A homomorphism of unital algebras preserves the two structures

$$
\varphi(1)=\tilde{1}, \quad \varphi\left(U_{x} y\right)=\tilde{U}_{\varphi(x)} \varphi(y) .
$$

Necessarily such a map preserves squares $\varphi\left(x^{2}\right)=\varphi\left(U_{x} 1\right)=\tilde{U}_{\varphi(x)} \varphi(1)=\tilde{U}_{\varphi(x)} \tilde{1}=\varphi(x)^{2}$. Thus we have a natural correspondence between unital quadratic algebras and quadratic algebras with unit.

In any quadratic algebra $\mathfrak{A}$ we can introduce a cubing operation

(1.4) $x^{3}=U_{x} x$

and this has a natural extension to any $\mathfrak{A}_{\Omega}$. Conversely, given squaring and cubing operations (the latter a homogeneous map of degree 3 ) in $\mathfrak{A}$ and all its extensions (or, equivalently, given the linearizations of the cubing operation), we can introduce a $U$-operator

(1.5) $U_{x} y=\left.\partial_{y} x^{3}\right|_{x}-x^{2} \circ y$ where $(x+\lambda y)^{3}=x^{3}+\left.\lambda \partial_{y} x^{3}\right|_{x}+\left.\lambda^{2} \partial_{x} y^{3}\right|_{y}+\lambda^{3} y^{3}$ in $\mathfrak{A}_{\Omega}, \Omega=\Phi[\lambda]$.

If we start with a cubing operation given by (1.4) our new $U$-operator $\widetilde{U}_{x}$ defined by (1.5) may differ from the original; they coincide if and only if (1.6) $\left\{\begin{array}{lll}x & x & y\end{array}\right\}=x^{2} \circ y$ 
since $\tilde{U}_{x} y=\left.\partial_{y} x^{3}\right|_{x}-x^{2} \circ y=U_{x} y+U_{x, y} x-x^{2} \circ y$. Conversely, if we start with a $U$-operator given by (1.5) the cube defined by (1.4) coincides with the original if and only if

(1.7) $x^{2} \circ x=2 x^{3}$

since $x^{\tilde{3}}=U_{x} x=\left.\partial_{x} x^{3}\right|_{x}-x^{2} \circ x=3 x^{3}-x^{2} \circ x$ by Euler's differential equation for homogeneous functions. One can also easily find conditions that an element $c$ satisfies (1.1)-(1.3).

THEOREM 1. If $\mathfrak{A}$ and $\mathfrak{\mathscr { U }}$ are quadratic algebras satisfying $\{x \quad x \quad y\}=x^{2} \circ y$ and $\varphi: \mathfrak{A} \rightarrow \mathfrak{A}$ a linear map such that

(i) $\varphi\left(x^{2}\right)=\varphi(x)^{2}$,

(ii) $\varphi\left(x^{3}\right)=\varphi(x)^{3}$

hold for all scalar extensions, then $\varphi$ is a homomorphism of quadratic algebras.

Proof. Our hypothesis on $\mathfrak{A}$ and $\mathfrak{A}$ guarantees that the $U$-operators are built up from the cubes (1.4) and the squares by the formula (1.5). Since (i) implies $\varphi\left(x^{2} \circ y\right)$ $=\varphi(x)^{\tilde{2}} \circ \varphi(y)$ and (ii) and its linearization imply $\varphi\left(\left.\partial_{y} x^{3}\right|_{x}\right)=\left.\partial_{y} \varphi\left(x^{3}\right)\right|_{x}=\left.\partial_{y} \varphi(x)^{\tilde{3}}\right|_{x}=$ $\left.\partial_{\varphi(y)} \tilde{x}^{\tilde{3}}\right|_{\varphi(x)}$, we see $\varphi\left(U_{x} y\right)=\tilde{U}_{\varphi(x)} \varphi(y)$.

Under certain conditions the requirement that (ii) hold in all extensions can be dropped (e.g. [1]). As usual, relations (i)-(ii) will hold for all extensions if $\Phi$ is a field with three or more elements. It is not true in general: if $\Phi=Z_{2}$ and $\mathfrak{A}, \tilde{\mathfrak{A}}$ are Boolean (associative) algebras with $x^{2}=x, U_{x} y=x y x=x^{2} y=x y=y x$ then any linear map satisfies (i) and (ii) since $\varphi\left(x^{3}\right)=\varphi\left(x^{2}\right)=\varphi(x)$ and $\varphi(x)^{\tilde{3}}=\varphi(x)^{\tilde{2}}=\varphi(x)$, but not all linear maps need be homomorphisms.

A unital quadratic Jordan algebra is a quadratic algebra $\Im$ with an element 1 satisfying

(UQJ 1) $U_{1}=I$,

(UQJ 2) $U_{U(x) y}=U_{x} U_{y} U_{x}$

(UQJ 3) $U_{x} V_{y, x}=V_{x, y} U_{x}$,

and such that these hold for all scalar extensions (equivalently, such that the axioms can be linearized). Such an algebra satisfies $(1.1)^{\prime},(1.2)^{\prime}$, so deserves the adjective "unital". It also satisfies (1.5)-(1.7), so $\Im$ is defined either by the $U$-structure or by the cubing structure.

An important kind of unital quadratic Jordan algebra is the algebra $\mathfrak{Q}^{+}$obtained from a unital associative algebra $\mathfrak{A}$ by taking $1=1$ and $U_{x} y=x y x$. Any unital (Jordan) subalgebra of such an algebra is called a special Jordan algebra.

If $\Im$ is a subspace of a unital associative algebra which contains 1 and is closed under squares and cubes, and such that it remains closed under all scalar extensions, then $\Im$ is a special Jordan subalgebra: the fact that $\Im$ is closed under cubes in all extensions guarantees $\mathfrak{I}$ is closed under the linearizations of the cubing operation, so (1.5) shows that $\Im$ is closed under the product $U_{x} y$. (Note that any algebra $\mathfrak{A}^{+}$satisfies (1.6).) 
Again, the closure under scalar extensions is not automatic: if $\Phi=Z_{2}$ and $\mathfrak{A}$ is Boolean then $x^{3}=x^{2}=x$, so any subspace is closed under squares and cubes.

2. The main calculations. In this section we prove a quadratic analogue of Theorem 1.

THEOREM 2. If $\mathfrak{A}$ is a unital quadratic algebra satisfying $\left\{\begin{array}{lll}x & x & y\end{array}\right\}=x^{2} \circ y$, and $\tilde{\mathfrak{A}}$ is a unital associative algebra, then any quadratic map $\varphi: \mathfrak{A} \rightarrow \tilde{\mathfrak{A}}$ such that

$$
\begin{aligned}
\varphi(1) & =1, \\
\varphi\left(x^{2}\right) & =\varphi(x)^{2}, \\
\varphi\left(x^{3}\right) & =\varphi(x)^{3}, \\
\varphi\left(U_{x} y, x\right) & =\varphi(x) \psi(y, x)=\psi(x, y) \varphi(x)
\end{aligned}
$$

holds for all scalar extensions, where $\varphi(x, y)=\varphi(x+y)-\varphi(x)-\varphi(y), \psi(x)=\varphi(x, 1)$, $\psi(x, y)=\psi(x) \psi(y)-\varphi(x, y)$, necessarily satisfies

$$
\varphi\left(U_{x} y\right)=\varphi(x) \varphi(y) \varphi(x) .
$$

For the proof we need a lemma, which we will prove in a generality suitable for later applications (including representation theory).

LEMMA 1. If $\mathfrak{A}$ is a quadratic algebra satisfying $\left\{\begin{array}{lll}x & x & y\end{array}\right\}=x^{2} \circ y, \tilde{\mathfrak{A}}$ an associative algebra, and $\varphi, \psi$ quadratic and linear maps respectively from $\mathfrak{A}$ to $\mathfrak{A}$ which satisfy

(2.3) $\varphi\left(x^{2}\right)=\varphi(x)^{2}$,

(2.4) $\psi\left(x^{2}\right)=\psi(x, x)$,

(2.5) $\psi\left(x^{3}\right)=\psi\left(x^{2}, x\right)=\psi\left(x, x^{2}\right)$,

(2.6) $\varphi\left(x^{2}, x\right)=\varphi(x) \psi(x)=\psi(x) \varphi(x)$,

(2.7) $\varphi(x \circ y, x)=\varphi(x) \psi(y)+\psi(y) \varphi(x)$,

(2.8) $\varphi\left(U_{x} y, x\right)=\varphi(x) \psi(y, x)=\psi(x, y) \varphi(x)$,

for all scalar extensions, where $\psi(x, y)=\psi(x) \psi(y)-\varphi(x, y)$, then $\varphi$ and $\psi$ also satisfy

(2.9) $2_{\varphi}(x)=\psi(x)^{2}-\psi\left(x^{2}\right)$,

(2.10) $\varphi\left(x^{2}, y\right)=\psi(x) \varphi(x, y)-\varphi(x) \psi(y)=\varphi(x, y) \psi(x)-\psi(y) \varphi(x)$,

(2.11) $\psi\left(U_{x} y\right)=\psi(x) \psi(y, x)-\psi(y) \varphi(x)=\psi(x, y) \psi(x)-\varphi(x) \psi(y)$,

(2.12) $\varphi\left(x^{3}, x\right)=\varphi(x) \psi\left(x^{2}\right)=\psi\left(x^{2}\right) \varphi(x)$,

(2.13) $\varphi\left(x^{3}, y\right)=\varphi(x, y) \psi\left(x^{2}\right)-\psi(y, x) \varphi(x)=\psi\left(x^{2}\right) \varphi(y, x)-\varphi(x) \psi(x, y)$

$=\varphi\left(x^{2}, y\right) \psi(x)-\varphi(x, y) \varphi(x)=\psi(x) \varphi\left(x^{2}, y\right)-\varphi(x) \varphi(x, y)$,

(2.14) $\varphi\left(U_{x} z, y\right)=\varphi(x, y) \psi(z, x)-\psi(y, z) \varphi(x)=\psi(x, z) \varphi(x, y)-\varphi(x) \psi(z, y)$,

(2.15) $\varphi\left(x^{3}, x^{2}\right)=\psi(x) \varphi\left(x^{2}\right)=\varphi\left(x^{2}\right) \psi(x)$,

(2.16) $\psi\left(x^{4}\right)=\psi\left(x, x^{3}\right)=\psi\left(x^{3}, x\right)$ for $x^{4}=\left(x^{2}\right)^{2}$.

Proof. (2.9) is just (2.4) since $\varphi(x, x)=2 \varphi(x)$. Linearizing (2.6), which is possible by our hypothesis that (2.3)-(2.8) hold for all extensions, gives $\varphi\left(x^{2}, y\right)+\varphi(x \circ y, x)$ $=\psi(y) \varphi(x)+\psi(x) \varphi(x, y)=\varphi(x) \psi(y)+\varphi(x, y) \psi(x)$, so (2.10) results by subtracting (2.7). 
Linearizing (2.5) gives $0=\psi\left(U_{x} y+x^{2} \circ y\right)-\left[\psi\left(x^{2}\right) \psi(y)-\varphi\left(x^{2}, y\right)\right]-[\psi(x \circ y) \psi(x)$ $-\varphi(x \circ y, x)]=\psi\left(x \circ(x \circ y)-U_{x} y\right)-\psi(x \circ y) \psi(x)+\varphi(x \circ y, x)-\left[\psi(x)^{2}-2 \varphi(x)\right] \psi(y)+$ $[\psi(x) \varphi(x, y)-\varphi(x) \psi(y)]$ (by (2.9), (2.10), and the fact that by linearizing our hypothesis on $\mathfrak{A}$ we get $\left.2 U_{x}=V_{x}^{2}-V_{x^{2}}\right)=\psi(x) \psi(x \circ y)-\varphi(x \circ y, x)-\psi\left(U_{x} y\right)-$ $\psi(x)[\psi(x) \psi(y)-\varphi(x, y)]+\varphi(x) \psi(y)$ (using linearized $(2.9))=\psi(x) \psi(y, x)-\psi(y) \varphi(x)$ $-\psi\left(U_{x} y\right)$ (by linearized (2.4), and (2.7)). Similarly we obtain the dual, so (2.11) holds.

(2.12) results from (2.8) by setting $y=x$ and using (2.4).

To obtain (2.13), linearize (2.12): $\varphi\left(x^{3}, y\right)=\varphi\left(U_{x} y, x\right)-\varphi(x \circ(x \circ y), x)$ $+\varphi(x) \psi(x \circ y)+\varphi(x, y) \psi\left(x^{2}\right)$ (by our hypothesis on $\mathfrak{A}$ again) $=\psi(x, y) \varphi(x)$ $-\psi(x \circ y) \varphi(x)+\varphi(x, y) \psi\left(x^{2}\right)$ (by (2.8), (2.7)) $=\varphi(x, y) \psi\left(x^{2}\right)-\psi(y, x) \varphi(x)$ (linearized (2.4)), and dually. For the other part of (2.13), note $\varphi\left(x^{2}, y\right) \psi(x)-\varphi(x, y) \varphi(x)$ $=[\varphi(x, y) \psi(x)-\psi(y) \varphi(x)] \psi(x)-\varphi(x, y) \varphi(x) \quad($ by $\quad(2.10))=\varphi(x, y)\left[\psi(x)^{2}-\varphi(x)\right]-$ $\psi(y) \psi(x) \varphi(x)($ by $(2.6))=\varphi(x, y)\left[\psi\left(x^{2}\right)+\varphi(x)\right]-\psi(y) \psi(x) \varphi(x)($ by $(2.9))=\varphi(x, y) \psi\left(x^{2}\right)$ $-\psi(y, x) \varphi(x)$.

Linearizing (2.13) gives $\varphi\left(U_{x} z, y\right)-\varphi(x, y) \psi(z, x)+\psi(y, z) \varphi(x)=-\varphi\left(x^{2} \circ z, y\right)$ $+\varphi(x, y) \psi(x, z)+\varphi(z, y) \psi\left(x^{2}\right)-\psi(y, x) \varphi(x, z)$ (using linearized (2.4))

$=-\left[\varphi\left(x^{2}, y\right) \psi(z)+\varphi(z, y) \psi\left(x^{2}\right)-\psi(y) \varphi\left(x^{2}, z\right)\right]+\varphi(x, y) \psi(x, z)+\varphi(z, y) \psi\left(x^{2}\right)$

$-[\psi(y) \psi(x)-\varphi(x, y)] \varphi(x, z) \quad$ (by linearized $\quad(2.10))=\varphi(x, y)[\psi(x, z)+\varphi(x, z)]$ $+\psi(y)\left[\varphi\left(x^{2}, z\right)-\psi(x) \varphi(x, z)\right]-\varphi\left(x^{2}, y\right) \psi(z)=\left[\varphi(x, y) \psi(x)-\varphi\left(x^{2}, y\right)\right] \psi(z)$

$-\psi(y)[\varphi(x) \psi(z)]$ (by (2.10)) $=0$ by (2.10) again. Similarly for the other half of (2.14)

Setting $y=x^{2}$ in (2.13) gives $\varphi\left(x^{3}, x^{2}\right)=\varphi\left(x, x^{2}\right) \psi\left(x^{2}\right)-\psi\left(x^{2}, x\right) \varphi(x)=\varphi(x) \psi(x) \psi\left(x^{2}\right)$ $-\psi\left(x^{2}, x\right) \varphi(x)$ (by $\left.(2.6)\right)=\left[\psi\left(x^{2}\right) \psi(x)-\psi\left(x^{2}, x\right)\right] \varphi(x)$ (commutativity follows from (2.6), (2.9)) $=\varphi\left(x^{2}, x\right) \varphi(x)=\varphi(x) \psi(x) \varphi(x)$ (by (2.6)), so from (2.3) we get (2.15).

Finally, for (2.16) $\psi\left(x^{4}\right)=\psi\left(x^{2}\right)^{2}-2 \varphi\left(x^{2}\right) \quad$ (by $\left.(2.9)\right)=\psi\left(x^{2}\right)\left[\psi(x)^{2}-2 \varphi(x)\right]$ $-\left[\psi(x)^{2}-\psi\left(x^{2}\right)\right] \varphi(x)$ (by (2.9), (2.3)) $=\psi\left(x^{2}\right) \psi(x)^{2}-\psi\left(x^{2}\right) \varphi(x)-\varphi\left(x^{2}, x\right) \psi(x)$ (by (2.6)) $=\psi\left(x^{2}, x\right) \psi(x)-\psi\left(x^{2}\right) \varphi(x)=\psi\left(x^{3}\right) \psi(x)-\varphi\left(x^{3}, x\right)$ (by $\left.(2.5),(2.12)\right)=\psi\left(x^{3}, x\right)$.

Proof of Theorem 2. Linearizing (2.1.iii) gives us a relation

$$
\begin{aligned}
& \varphi\left(x^{3}, U_{y} x+y^{2} \circ x\right)+\varphi\left(U_{x} y+x^{2} \circ y\right) \\
& =\varphi(x)^{2} \varphi(y)+\varphi(x) \varphi(y) \varphi(x)+\varphi(y) \varphi(x)^{2}+\varphi(x, y)^{2} \varphi(x) \\
& +\varphi(x, y) \varphi(x) \varphi(x, y)+\varphi(x) \varphi(x, y)^{2}
\end{aligned}
$$

since our assumption on $\mathfrak{A}$ guarantees $\left.\partial_{y} x^{3}\right|_{x}=U_{x} y+x^{2} \circ y$. Similarly, linearizing (ii) gives

$$
\varphi(x \circ y)+\varphi\left(x^{2}, y^{2}\right)=\varphi(x) \varphi(y)+\varphi(y) \varphi(x)+\varphi(x, y)^{2} .
$$

Putting this (with $x$ replaced by $x^{2}$ ) into the previous relation and using (ii) yields

$$
\begin{aligned}
& \varphi\left(U_{x} y\right)-\varphi(x) \varphi(y) \varphi(x) \\
&=-\varphi\left(x^{3}, U_{y} x\right)-\varphi\left(x^{3}, y^{2} \circ x\right)-\varphi\left(U_{x} y, x^{2} \circ y\right)+\varphi\left(x^{4}, y^{2}\right)-\varphi\left(x^{2}, y\right)^{2} \\
& \\
&+\varphi(x, y)^{2} \varphi(x)+\varphi(x, y) \varphi(x) \varphi(x, y)+\varphi(x, y)^{2} \varphi(x) .
\end{aligned}
$$


Thus the identity (2.2) will follow if we can prove

(2.17) $\varphi\left(x^{3}, x \circ z\right)=\varphi(x) \varphi\left(x^{2}, z\right)+\varphi\left(x^{2}, z\right) \varphi(x)$,

(2.18) $\varphi\left(x^{3}, U_{x} y\right)=\varphi\left(x^{4}, y^{2}\right)-\varphi\left(x^{2}, y\right)^{2}+\varphi(x, y) \varphi(x) \varphi(x, y)$,

(2.19) $\varphi\left(U_{x} y, x^{2} \circ y\right)=\varphi(x)\left[\varphi(x, y)^{2}-\varphi\left(x^{2}, y^{2}\right)\right]+\left[\varphi(x, y)^{2}-\varphi\left(x^{2}, y^{2}\right)\right] \varphi(x)$

(we actually only need (2.17) for $z=y^{2}$ ).

To prove these we will want the formulas (2.3)-(2.16), so we first check that the hypotheses of Lemma 1 are met. (2.3) and (2.8) are just our assumptions (ii) and (iv). (2.6) follows by setting $y=1$ in (iv) since $\psi(x, 1)=\psi(1, x)=\psi(x)$ because $\psi(1)=\varphi(1,1)=2 \varphi(1)=2$ from (i). For (2.4), linearize (2.6) to get $\varphi\left(x^{2}, 1\right)+2 \varphi(x, x)$ $=\varphi(x) \psi(1)+\varphi(x, 1) \psi(x)$, hence $\psi\left(x^{2}\right)+4 \varphi(x)=2 \varphi(x)+\psi(x)^{2}$. To obtain (2.5), put $y=x$ in (iv) to get $\varphi\left(x^{3}, x\right)=\varphi(x) \psi\left(x^{2}\right)$ by (2.4), then linearize to get $\varphi\left(x^{3}, 1\right)+$ $3 \varphi\left(x^{2}, x\right)=2 \varphi(x) \psi(x)+\varphi(x, 1) \psi\left(x^{2}\right)$ or $\psi\left(x^{3}\right)=\psi(x) \psi\left(x^{2}\right)-\varphi\left(x^{2}, x\right)($ by $(2.6))=\psi\left(x, x^{2}\right)$ $=\psi\left(x^{2}, x\right)\left(\right.$ since $\psi(x)$ commutes with $\left.\psi\left(x^{2}\right)\right)$.

The most difficult is (2.7): we linearize (iv) to obtain $\varphi\left(U_{x, z} x, 1\right)+\varphi\left(U_{x, 1} x, z\right)$ $+\varphi\left(U_{z, 1} x, x\right)=\varphi(x, 1) \psi(x, z)+\varphi(x, z) \psi(x, 1)+\varphi(z, 1) \psi(x, x)$ or $\varphi(z \circ x, x)$ $=-\psi\left(x^{2} \circ z\right)-2 \varphi\left(x^{2}, z\right)+\psi(x) \psi(x, z)+\varphi(x, z) \psi(x)+\psi(z) \psi\left(x^{2}\right)=-\left[\psi\left(x^{2}\right) \psi(z)\right.$ $\left.+\psi(z) \psi\left(x^{2}\right)\right]+\psi(x)^{2} \psi(z)-\psi(x) \varphi(x, z)+\varphi(x, z) \psi(x)+\psi(z) \psi\left(x^{2}\right)$ (by linearized (2.4)) $=\left[\psi(x)^{2}-\psi\left(x^{2}\right)\right] \psi(z)+[\varphi(x, z) \psi(x)-\psi(x) \varphi(x, z)]=2 \varphi(x) \psi(z)-[\varphi(x) \psi(z)-\psi(z) \varphi(x)]$ (by linearized (2.6), (2.4)) $=\varphi(x) \psi(z)+\psi(z) \varphi(x)$ as desired.

To verify (2.17), $\varphi\left(x^{3}, x \circ z\right)=\varphi\left(x^{2}, x \circ z\right) \psi(x)-\varphi(x, x \circ z) \varphi(x) \quad$ (by (2.13)) $=[\varphi(x) \varphi(x, z)+\varphi(x, z) \varphi(x)] \psi(x)-[\varphi(x) \psi(z)+\psi(z) \varphi(x)] \varphi(x)$ (by linearized (2.3), (2.7)) $=\varphi(x)[\varphi(x, z) \psi(x)-\psi(z) \varphi(x)]+[\varphi(x, z) \psi(x)-\psi(z) \varphi(x)] \psi(x) \quad$ (by $=\varphi(x) \varphi\left(x^{2}, z\right)+\varphi\left(x^{2}, z\right) \varphi(x)$ (by (2.10)).

For (2.18), $\varphi\left(x^{3}, U_{y} x\right)=\psi(y, x) \varphi\left(y, x^{3}\right)-\varphi(y) \psi\left(x, x^{3}\right)($ by $(2.14))=\psi(y) \psi(x) \varphi\left(y, x^{3}\right)$ $-\varphi(x, y) \varphi\left(x^{3}, y\right)-\varphi(y) \psi\left(x^{4}\right)$ (by $\left.(2.16)\right)=\psi(y)\left[\psi(x) \varphi\left(y, x^{3}\right)-\varphi\left(x^{4}, y\right)\right]$

$-\varphi(x, y)\left[\psi(x) \varphi\left(x^{2}, y\right)-\varphi(x) \varphi(x, y)\right]+\varphi\left(x^{4}, y^{2}\right)$ (by (2.10), (2.13))

$=\psi(y)\left[\psi(x)\left\{\psi(x) \varphi\left(x^{2}, y\right)-\varphi(x) \varphi(x, y)\right\}-\left\{\psi\left(x^{2}\right) \varphi\left(x^{2}, y\right)-\varphi\left(x^{2}\right) \psi(y)\right\}\right]$

$+\varphi(x, y) \varphi(x) \varphi(x, y)+\varphi\left(x^{4}, y^{2}\right)-\left[\varphi\left(x^{2}, y\right)+\psi(y) \varphi(x)\right] \varphi\left(x^{2}, y\right)$ (by (2.13), (2.10)) $=\varphi\left(x^{4}, y^{2}\right)-\varphi\left(x^{2}, y\right)^{2}+\varphi(x, y) \varphi(x) \varphi(x, y)+\psi(y) \varphi(x)\left[2 \varphi\left(x^{2}, y\right)-\psi(x) \varphi(x, y)+\varphi(x) \psi(y)\right.$ $\left.-\varphi\left(x^{2}, y\right)\right]($ by $(2.9))=\varphi\left(x^{4}, y^{2}\right)-\varphi\left(x^{2}, y\right)^{2}+\varphi(x, y) \varphi(x) \varphi(x, y)$ as desired.

Before establishing (2.19) we show

$$
\psi(x, y) \psi(y, x)=\varphi(x, y)^{2}-\varphi\left(x^{2}, y^{2}\right)+\psi\left(U_{x} y^{2}\right) .
$$

We have $\psi(x, y) \psi(y, x)=\varphi(x, y)^{2}+\psi(x) \psi(y)^{2} \psi(x)-\psi(x) \psi(y) \varphi(x, y)-\varphi(x, y) \psi(y) \psi(x)$ $=\varphi(x, y)^{2}+\psi(x)\left[2 \varphi(y)+\psi\left(y^{2}\right)\right] \psi(x)-\psi(x)\left[\varphi\left(x, y^{2}\right)+\varphi(y) \psi(x)\right]$

$-\left[\varphi\left(x, y^{2}\right)+\psi(x) \varphi(y)\right] \psi(x)$ (by (2.9), (2.10)) $=\varphi(x, y)^{2}+\psi(x) \psi\left(y^{2}\right) \psi(x)-\psi(x) \varphi\left(x, y^{2}\right)$ $-\left[\varphi\left(x^{2}, y^{2}\right)+\psi\left(y^{2}\right) \varphi(x)\right]($ by $(2.10))=\varphi(x, y)^{2}-\varphi\left(x^{2}, y^{2}\right)+\psi\left(U_{x} y^{2}\right)$ (by (2.11)). Next,

$$
\begin{aligned}
& \psi(x) \psi(x, z)-\varphi(x) \psi(z)=\psi\left(x^{2}, z\right), \\
& \psi(z, x) \psi(x)-\psi(z) \varphi(x)=\psi\left(z, x^{2}\right),
\end{aligned}
$$

since, for example, $\psi(x) \psi(x, z)-\varphi(x) \psi(z)=\left[\psi(x)^{2}-\varphi(x)\right] \psi(z)-\psi(x) \varphi(x, z)$ $=\left[\psi\left(x^{2}\right)+\varphi(x)\right] \psi(z)-\varphi\left(x^{2}, z\right)-\varphi(x) \psi(z)($ by $(2.9),(2.10))=\psi\left(x^{2}, z\right)$. 
Now by (2.14)

$$
\varphi\left(U_{x} y, x^{2} \circ y\right)=\varphi\left(x, x^{2} \circ y\right) \psi(z, x)-\psi\left(x^{2} \circ y, y\right) \varphi(x)
$$

where

$$
\psi\left(x^{2} \circ y, y\right)=\psi\left(U_{y} x^{2}\right)+\psi\left(x^{2}, y^{2}\right)
$$

since $\psi\left(x^{2} \circ y, y\right)=\left[\psi\left(x^{2}, y\right)+\psi\left(y, x^{2}\right)\right] \psi(y)-\varphi\left(x^{2} \circ y, y\right)$ (by (2.4)) $=\left[\psi\left(y, x^{2}\right) \psi(y)\right.$ $\left.-\varphi(y) \psi\left(x^{2}\right)\right]+\left[\psi\left(x^{2}, y\right) \psi(y)-\psi\left(x^{2}\right) \varphi(y)\right]$ and we can apply (2.11), (2.21), where

$$
\varphi\left(x, x^{2} \circ y\right)=\varphi(x) \psi(x, y)+\psi(y, x) \varphi(x)
$$

since $\varphi\left(x, x^{2} \circ y\right)=-\varphi\left(x^{2}, x \circ y\right)+\varphi\left(x, x^{2}\right) \psi(y)+\psi(y) \varphi\left(x, x^{2}\right) \quad$ (linearizing (2.7)) $=-\varphi(x) \varphi(x, y)-\varphi(x, y) \varphi(x)+\varphi(x) \psi(x) \psi(y)+\psi(y) \psi(x) \varphi(x)$ (linearizing (2.3), (2.6)) $=\varphi(x) \psi(x, y)+\psi(y, x) \varphi(x)$, so that

$$
\varphi\left(x, x^{2} \circ y\right) \psi(y, x)=\varphi(x) \psi(x, y) \psi(y, x)+\psi(y, x) \psi(x, y) \varphi(x)
$$

by (2.8); and where

$$
\varphi(x) \psi\left(U_{x} y^{2}\right)=\psi\left(x^{2}, y^{2}\right) \varphi(x)
$$

since $\varphi(x) \psi\left(U_{x} y^{2}\right)=\varphi(x)\left[\psi(x) \psi\left(y^{2}, x\right)-\psi\left(y^{2}\right) \varphi(x)\right]$ (by $\left.\quad(2.11)\right)=\left[\psi(x) \psi\left(x, y^{2}\right)\right.$ $\left.-\varphi(x) \psi\left(y^{2}\right)\right] \varphi(x)$ (by (2.6), (2.8)) and we can apply (2.21). Putting these all together by means of (2.20) we arrive at (2.19). This completes the proof of Theorem 2.

3. Alternate axiomatization. In this section we give an alternate axiomatization of unital quadratic Jordan algebras in terms of identities which involve only a single variable $x$. This will prove useful in the next two sections when we verify that certain algebras satisfy our axioms; when we attempt to prove something about an operator such as $U_{x} U_{y} U_{x}$ the $x$ 's and $y$ 's get all tangled up in each other, whereas in an expression like $U_{x} U_{x} U_{x}$ involving $x$ alone the terms all commute smoothly.

THEOREM 3. A unital quadratic algebra is a unital quadratic Jordan algebra if and only if the identities

(UCJ 1) $U_{1}=I$,

(UCJ 2) $V_{x, x}=V_{x^{2}}$,

(UCJ 3) $U_{x} V_{x}=V_{x} U_{x}$

(UCJ 4) $U_{x^{2}}=U_{x}^{2}$,

(UCJ 5) $U_{x^{3}}=U_{x}^{3}$

hold for all scalar extensions.

Proof. The conditions are certainly necessary: UCJ 1 is just UQJ 1 , UCJ 3 is UQJ 3 with $y=1$, UCJ 4 is UQJ 2 with $y=1$, UCJ 5 is UQJ 2 with $y=x$, while we have noted before that UCJ 2 holds. The same goes for any extension. 
Conversely, we must prove UCJ 1-5 imply UQJ 1-3. Clearly UQJ 1 holds. To prove UQJ 3 we need a lemma.

LEMMA 2. If a quadratic algebra (not necessarily unital) satisfies

(3.1) $V_{x, x}=V_{x^{2}}$,

(3.2) $U_{x} V_{x}=V_{x} U_{x}$,

(3.3) $U_{x}\left(x^{2}\right)=\left(x^{2}\right)^{2}$,

(3.4) $U_{x^{2}}=U_{x}^{2}$,

for all scalar extensions, then it also satisfies

(3.5) $U_{x^{2}, x}=U_{x} V_{x}=V_{x} U_{x}$,

(3.6) $x \circ U_{y} x=y \circ U_{x} y$,

(3.7) $U_{x \circ y, x}=U_{x} V_{y}+V_{y} U_{x}$,

(3.8) $V_{x^{3}}=V_{x}\left\{V_{x^{2}}-U_{x}\right\}=V_{x, x^{2}}=V_{x^{2}, x}$,

(3.9) $U_{U(x) y, x}=U_{x} V_{y, x}=V_{x, y} U_{x}$.

Proof. For (3.5), $U_{x^{2}, x} y=\left\{x^{2} y x\right\}=\left(x^{2} \circ y\right) \circ x-\left\{y x^{2} x\right\} \quad$ (linearizing 3.1) $=x^{2} \circ(y \circ x)-U_{x, y} x^{2}$ (since $V_{x}$ commutes with $V_{x^{2}}=V_{x, x}=V_{x}^{2}-U_{x, x}$ by (3.1), its linearization, and (3.2)) $=U_{x}(x \circ y)$ (by linearized (3.3)) $=U_{x} V_{x} y$.

For (3.6), $x \circ U_{y} x=V_{x} U_{y} x=\left\{-V_{y} U_{x, y}+U_{x, y} V_{y}+U_{y} V_{x}\right\} x$ (linearized (3.2)) $=-y \circ\left\{\begin{array}{lll}x & y\end{array}\right\}+U_{x, y}(x \circ y)+2 U_{y} x^{2}=-y \circ\left(y \circ x^{2}\right)+2 U_{y} x^{2}+U_{x, y}(x \circ y)$ (by (3.1)) $=-y^{2} \circ x^{2}+U_{x, y}(x \circ y)$, which is symmetric in $x$ and $y$.

For (3.7), $\left\{V_{y} U_{x}+U_{x} V_{y}\right\} z=y \circ U_{x} z+U_{x}(y \circ z)=x \circ U_{y, z} x-z \circ U_{x} y+U_{x} V_{z} y$ (linearizing (3.6)) $=x \circ\{y x z\}+\left\{U_{x} V_{z}-V_{z} U_{x}\right\} y=x \circ\{(y \circ x) \circ z\}-x \circ\{x y z\}+$ $\left\{V_{x} U_{x, z}-U_{x, z} V_{x}\right\} y$ (linearizing (3.1), (3.2)) $=x \circ\{z \circ(x \circ y)\}-\{x x \circ y z\}=\{x z x \circ y\}$ (linearized (3.1)) $=U_{x_{0} y, x}$.

We can reinterpret this to get (3.8): $V_{x^{3}} y=y \circ x^{3}=V_{y} U_{x} x=\left\{U_{x \circ y, x}-U_{x} V_{y}\right\} x$ $=\{x \circ y \quad x \quad x\}-U_{x}(x \circ y)=\left\{V_{x, x} V_{x}-U_{x} V_{x}\right\} y$ and the rest follows from (3.1) and (3.5).

Finally, for (3.9) we need only establish the second equality, since then $U_{U(x) y, x} z=\left\{U_{x} y z x\right\}=V_{x, z} U_{x} y=U_{x} V_{z, x} y=U_{x} V_{y, x} \dot{z}$ gives the other equality. But $U_{x} U_{x, y}+U_{x, y} U_{x}=U_{x^{2}, x \circ y} \quad$ (linearizing (3.4)) $=-U_{x \circ(x \circ y), x}+U_{x} V_{x \circ y}+U_{x \circ y, x} V_{x}$ (linearizing (3.5)) $=-V_{x \circ y} U_{x}+\left\{U_{x} V_{y}+V_{y} U_{x}\right\} V_{x}$ (by (3.7)) $=-\left\{V_{x, y}+V_{y, x}\right\} U_{x}+$ $U_{x}\left\{V_{y, x}+U_{x, y}\right\}+V_{y} V_{x} U_{x}$ (by (3.2) and linearized (3.1)) $=U_{x} V_{y, x}-V_{x, y} U_{x}+$ $U_{x, y} U_{x}+U_{x} U_{x, y}$ so $U_{x} V_{y, x}-V_{x, y} U_{x}=0$.

To return to the proof of the theorem, in our case (3.1), (3.2), (3.4) are just the axioms UCJ 2, UCJ 3, UCJ 4, and (3.3) is just UCJ 4 applied to the element 1. Thus the lemma applies, and we conclude by (3.9) that UQJ 3 holds.

To prove UQJ 2 it suffices to meet the conditions of Theorem 2 for $\varphi(x)=U_{x}$, $\psi(x)=V_{x}$ (note $\psi(x, y)=V_{x} V_{y}-U_{x, y}=V_{x, y}$ by linearized UCJ 2). Parts (i), (ii), (iii) of (2.1) are just UCJ 1, UCJ 4, UCJ 5, and (iv) is the axiom UQJ 3 we have just finished establishing. Thus Theorem 2 is applicable, and by (2.2) we conclude $U_{U(x) y}=U_{x} U_{y} U_{x}$, which is UQJ 2.

Some results characterizing Jordan algebras in terms of UCJ 4 and UCJ 5 (as well as in terms of UQJ 2) were first obtained by M. Koecher (see [2]). 
4. Quadratic Jordan algebras without unit. Whatever else general quadratic Jordan algebras should be, they should be precisely those subspaces $\Im$ of unital quadratic Jordan algebras $\Im^{\prime}$ which are closed under $x^{2}$ and $U_{x} y$ (see [3, p. 1072]). We hope to capture this property in the following axiomatic description. A quadratic Jordan algebra is a quadratic algebra $\Im=\left(X, U,{ }^{2}\right)$ where the compositions $x^{2}$ and $U_{x} y$ are related by

(QJ 1) $V_{x, x}=V_{x^{2}}$

(QJ 2) $U_{x} V_{x}=V_{x} U_{x}$,

(QJ 3) $U_{x}\left(x^{2}\right)=\left(x^{2}\right)^{2}$,

(QJ 4) $U_{x} U_{y}\left(x^{2}\right)=\left(U_{x} y\right)^{2}$,

(QJ 5) $U_{x^{2}}=U_{x}^{2}$,

(QJ 6) $U_{U(x) y}=U_{x} U_{y} U_{x}$,

and such that these remain valid under all scalar extensions (equivalently, their linearizations hold in $\Im$ ). For example, if $\Im$ is a subspace of an associative algebra closed under $x^{2}$ and $U_{x} y=x y x$ the relations QJ 1-6 hold by inspection of

$$
\begin{array}{ccc}
x x z+z x x & x x z x+x z x x & x^{4} \\
x y x^{2} y x & x x z x x & x y x z x y x .
\end{array}
$$

If the quadratic algebra $\Im$ is imbedded as a subalgebra of a unital Jordan algebra then QJ 1-6 hold in $\Im$ since they hold in $\Im^{\prime}$, and the same holds for any extension. Indeed, QJ 6 is the axiom UQJ 2, QJ 5 results by setting $y=1$ in QJ 6, QJ 4 and QJ 3 result by applying QJ 6 and QJ 5 to the element 1, QJ 2 results by setting $y=1$ in the axiom UQJ 2 since $V_{x}=V_{x, 1}=V_{1, x}$, and we have seen QJ 1 holds in any unital Jordan algebra.

It will be convenient to have a simpler axiomatization where conditions QJ 4 and 6 on the composition $U_{x} y$ are replaced by the analogous conditions on the cube $x^{3}$.

THEOREM 4. A quadratic algebra is a Jordan algebra if and only if the identities

(CJ 1) $V_{x, x}=V_{x^{2}}$

(CJ 2) $U_{x} V_{x}=V_{x} U_{x}$,

(CJ 3) $U_{x}\left(x^{2}\right)=\left(x^{2}\right)^{2}$,

(CJ 4) $\left(x^{3}\right)^{2}=\left(x^{2}\right)^{3}$,

(CJ 5) $U_{x^{2}}=U_{x}^{2}$

(CJ 6) $U_{x^{3}}=U_{x}^{3}$

hold for all scalar extensions.

Clearly CJ 1-6 hold in any quadratic Jordan algebra; CJ 6 results from QJ 6 by setting $y=x$, as does CJ 4 from QJ 4 using QJ $5\left(\left(x^{3}\right)^{2}=U_{x} U_{x} x^{2}=U_{x^{2}}\left(x^{2}\right)=\left(x^{2}\right)^{3}\right)$.

The justification for our axiomatization lies in

THEOREM 5. Any quadratic Jordan algebra $\Im$ can be imbedded as a subalgebra of the unital quadratic Jordan algebra $\mathfrak{\Im}^{\prime}=\Phi 1 \oplus \Im$ with unit $1^{\prime}=1 \oplus 0$ and U-operator

$$
U_{\alpha 1+x}^{\prime}(\beta 1+y)=\alpha^{2} \beta 1+\alpha^{2} y+2 \alpha \beta x+\alpha x \circ y+\beta x^{2}+U_{x} y \text {. }
$$


COROLLARY. $\Im$ is a quadratic Jordan algebra if and only if it is a subspace of some unital quadratic Jordan algebra $\mathfrak{F}^{\prime}$ closed under the compositions $x^{2}$ and $U_{x} y$.

Note that $\mathfrak{I}$ is indeed imbedded as a subalgebra of the $\mathfrak{I}^{\prime}$ constructed above: $x^{2 \prime}=U_{x}^{\prime} 1=x^{2}$ and $U_{x}^{\prime} y=U_{x} y$.

We prove Theorems 4 and 5 by showing the following conditions on an algebra $\Im$ are equivalent:

(I) $\Im$ satisfies QJ 1-6 for all extensions,

(II) Э satisfies CJ 1-6 for all extensions,

(III) $\Im^{\prime}$ defined by (4.1) is Jordan.

We have already remarked that III implies I and I implies II, so we need only show II implies III.

Thus we assume $\Im$ satisfies CJ 1-6 and must show $\mathfrak{\Im}^{\prime}$ satisfies UCJ $1-5$. The first step is to show that the hypotheses of Lemma 1 are met, so that we may use (2.3)-(2.16). Here $\varphi(x)=U_{x}, \psi(x)=V_{x}$, so $\psi(x, y)=V_{x} V_{y}-U_{x, y}=V_{x, y}$ by CJ 1 . The relations (2.3) and (2.4) are just CJ 5 and $C J 1$, and the relations (2.5)-(2.8) follow from Lemma 2 (which is applicable by CJ 1-5).

Now we use (2.3)-(2.16) and our axioms to establish UCJ 1-5. From (4.1) we see the following formulas for the operators $U_{x^{\prime}}^{\prime}$ and $V_{x^{\prime}}^{\prime}=V_{x^{\prime}, 1}^{\prime}$ in $\Im^{\prime}$ :

(4.3) $U_{1}^{\prime}=I, V_{1}^{\prime}=2 I$,

(4.4) $V_{x, 1}^{\prime}=V_{1, x}^{\prime}=U_{x, 1}^{\prime}=V_{x}^{\prime}$,

(4.5) $U_{x}^{\prime}=U_{x}, V_{x}^{\prime}=V_{x}$ on $\mathfrak{g}$ for $x$ in $\Im$,

(4.6) $U_{x}^{\prime} 1=x^{2}, V_{x}^{\prime} 1=2 x$ for $x$ in $\Im$,

(4.7) $U_{\alpha 1+x}^{\prime}=\alpha^{2} I+\alpha V_{x}^{\prime}+U_{x}^{\prime}$.

We use these to investigate UCJ 1-5. UCJ 1 follows from (4.3). For UCJ 2 we have

$$
\begin{aligned}
V_{\alpha 1+x, \alpha 1+x}^{\prime} & =2 \alpha^{2} I+2 \alpha V_{x}^{\prime}+V_{x, x}^{\prime}, \\
V_{(\alpha 1+x)^{2}}^{\prime} & =V_{\alpha^{2} 1+2 \alpha x+x^{2}}^{\prime}=2 \alpha^{2} I+2 \alpha V_{x}^{\prime}+V_{x^{2}}^{\prime 2}
\end{aligned}
$$

and we have $V_{x, x}^{\prime}=V_{x^{2}}^{\prime}$ on $\Im$ by CJ 1 and on $\Phi 1$ by $V_{x, x}^{\prime} 1=\left\{\begin{array}{lll}x & x & 1\end{array}\right\}=V_{x}^{\prime} x=2 x^{2}=$ $V_{x^{2}}^{\prime 2}$.

For UCJ 3 we have

$$
\begin{aligned}
& U_{\alpha 1+x}^{\prime} V_{\alpha 1+x}^{\prime}=2 \alpha^{3} I+3 \alpha^{2} V_{x}^{\prime}+\alpha\left\{2 U_{x}^{\prime}+V_{x}^{\prime 2}\right\}+U_{x}^{\prime} V_{x}^{\prime}, \\
& V_{\alpha 1+x}^{\prime} U_{\alpha 1+x}^{\prime}=2 \alpha^{3} I+3 \alpha^{2} V_{x}^{\prime}+\alpha\left\{2 U_{x}^{\prime}+V_{x}^{\prime 2}\right\}+V_{x}^{\prime} U_{x}^{\prime},
\end{aligned}
$$

where $U_{x}^{\prime} V_{x}^{\prime}=V_{x}^{\prime} U_{x}^{\prime}$ on $\Im$ by CJ 2 and on $\Phi 1$ by $U_{x}^{\prime} V_{x}^{\prime} 1=2 U_{x} x=x \circ x^{2}=V_{x}^{\prime} U_{x}^{\prime} 1$ from CJ 1 . We will use this commutativity from now on.

For UCJ 4 we have

$$
\begin{aligned}
& U_{(\alpha 1+x)^{2}}^{\prime}=U_{\alpha^{2} 1+2 a x+x^{2}}^{\prime}=\alpha^{4} I+2 \alpha^{3} V_{x}^{\prime}+\alpha^{2}\left\{V_{x^{2}}^{\prime}+4 U_{x}^{\prime}\right\}+2 \alpha U_{x^{2}, x}^{\prime}+U_{x^{2}}^{\prime} \text {, } \\
& U_{\alpha 1+x}^{\prime 2}=\left\{\alpha^{2} I+\alpha V_{x}^{\prime}+U_{x}^{\prime}\right\}^{2}=\alpha^{4} I+2 \alpha^{3} V_{x}^{\prime}+\alpha^{2}\left\{V_{x}^{\prime 2}+2 U_{x}^{\prime}\right\}+2 \alpha V_{x}^{\prime} U_{x}^{\prime}+U_{x}^{\prime 2} \text {, }
\end{aligned}
$$

where corresponding terms agree on $\Im$ by (2.9), (2.6), CJ 5 and on $\Phi 1$ by $2 U_{x}^{\prime} 1=2 x^{2}=2 x \circ x-2 x^{2}=\left\{V_{x}^{\prime 2}-V_{x^{2}}^{\prime}\right\} 1, U_{x^{2}, x}^{\prime} 1=x^{2} \circ x=V_{x} x^{2}=V_{x}^{\prime} U_{x}^{\prime} 1$, and CJ 3 . 
For UCJ 5 we have

$$
\begin{aligned}
U_{(\alpha 1+x)^{3}}^{\prime}= & U_{\alpha^{3} 1+3 \alpha^{2} x+3 \alpha x^{2}+x^{3}}^{\prime} \\
= & \alpha^{6} I+3 \alpha^{5} V_{x}^{\prime}+3 \alpha^{4}\left\{3 U_{x}^{\prime}+V_{x^{2}}^{\prime}\right\}+\alpha^{3}\left\{9 U_{x^{2}, x}^{\prime}+V_{x^{3}}^{\prime}\right\} \\
& +3 \alpha^{2}\left\{3 U_{x^{2}}^{\prime}+U_{x^{3}, x}^{\prime 3}\right\}+3 \alpha U_{x^{3}, x^{2}}^{\prime}+U_{x^{3}}^{\prime 3}, \\
U_{\alpha 1+x}^{\prime 3}= & \left\{\alpha^{2} I+\alpha V_{x}^{\prime}+U_{x}^{\prime}\right\}^{3} \\
= & \alpha^{6} I+3 \alpha^{5} V_{x}^{\prime}+3 \alpha^{4}\left\{U_{x}^{\prime}+V_{x}^{\prime 2}\right\}+\alpha^{3}\left\{6 V_{x}^{\prime} U_{x}^{\prime}+V_{x}^{\prime 3}\right\} \\
& +3 \alpha^{2}\left\{U_{x}^{\prime 2}+V_{x}^{\prime 2} U_{x}^{\prime}\right\}+3 \alpha V_{x}^{\prime} U_{x}^{\prime 2}+U_{x}^{\prime 3} .
\end{aligned}
$$

In view of our previous relations we need only prove

$$
\begin{aligned}
V_{x^{3}}^{\prime} & =V_{x}^{\prime 3}-3 V_{x}^{\prime} U_{x}^{\prime}, & U_{x^{3}, x}^{\prime} & =V_{x^{2}}^{\prime} U_{x}^{\prime}, \\
U_{x^{3}, x^{2}}^{\prime} & =V_{x}^{\prime} U_{x}^{\prime 2}, & U_{x^{3}}^{\prime} & =U_{x}^{\prime 3}
\end{aligned}
$$

and these hold on $\Im$ by (2.4)-(2.6), (2.12), (2.15), CJ 6 and on $\Phi 1$ by

$$
\begin{aligned}
V_{x^{3}}^{\prime} 1 & =2 x^{3}=4 x \circ x^{2}-3 x \circ x^{2}=\left\{V_{x}^{\prime 3}-3 V_{x}^{\prime} U_{x}^{\prime}\right\} 1, \\
U_{x^{3}, x}^{\prime} 1 & =x^{3} \circ x=V_{x} U_{x} x=U_{x} V_{x} x=U_{x}^{\prime} V_{x^{2}}^{\prime} 1, \\
U_{x^{3}, x^{2}}^{\prime} 1 & =x^{3} \circ x^{2}=V_{x^{2}} U_{x} x=U_{x} V_{x^{2}} x=U_{x} V_{x} x^{2}=U_{x}^{\prime} V_{x}^{\prime} U_{x}^{\prime} 1,
\end{aligned}
$$

and by $\mathrm{CJ} 4$.

5. Noncommutative Jordan algebras. In this section we show that with any noncommutative Jordan algebra $\mathfrak{A}$ we can associate in a natural way a quadratic Jordan algebra $\mathfrak{A}^{+}$, which coincides with the usual commutative Jordan algebra $\mathfrak{A}^{+}$in case $\frac{1}{2} \in \Phi$.

Recall [4] that a noncommutative Jordan algebra is a linear algebra $\mathfrak{A}$ in which for every $x$ the multiplication operators $L_{x}, R_{x}, L_{x^{2}}, R_{x^{2}}$ commute and such that the same holds for all scalar extensions. (This follows automatically if $\Phi$ is a field with more than two elements, in particular if the characteristic is $\neq 2$.) Then all the operators $L_{x^{n}}, R_{x^{m}}$ commute, and $\mathfrak{A}$ is strictly power-associative.

In any strictly power-associative linear algebra we have squaring and cubing operations

$$
x^{2}=x x, \quad x^{3}=x x^{2}=x^{2} x .
$$

From these we derive a $U$-operator $U_{x} y=\left.\partial_{y} x^{3}\right|_{x}-x^{2} \circ y=y x^{2}+x(x y+y x)-$ $\left(x^{2} y+y x^{2}\right)=x(x y+y x)-x^{2} y$ and dually,

$$
U_{x}=L_{x} V_{x}-L_{x^{2}}=R_{x} V_{x}-R_{x^{2}} \quad\left(V_{x}=L_{x}+R_{x}\right)
$$

(see [5, p. 90], [4, p. 1]). Note that $2 U_{x}=\left(L_{x}+R_{x}\right) V_{x}-\left(L_{x^{2}}+R_{x^{2}}\right)$, or

$$
2 U_{x}=V_{x}^{2}-V_{x^{2}}
$$

so if $\frac{1}{2} \in \Phi$ we see $U_{x}=2 L_{x}^{+2}-L_{x^{2}}^{+}=U_{x}^{+}$is the usual operator in the commutative algebra $\mathfrak{A}^{+}$(where $\left.L_{x}^{+} y=x \cdot y=\frac{1}{2}(x y+y x)=\frac{1}{2} x \circ y\right)$. One checks

$$
U_{x} x=x^{3}
$$


by direct computation or using (1.7) and $x \circ x^{2}=2 x^{3}$. As we have seen (or as one easily verifies directly)

$$
\left\{\begin{array}{lll}
x & x & y
\end{array}\right\}=x^{2} \circ y \quad \text { or } \quad V_{x, x}=V_{x^{2}}
$$

If $\mathfrak{A}$ has a unit then

$$
U_{1}=I, \quad V_{1}=2 I, \quad U_{x} 1=x^{2} .
$$

If the algebra we begin with is a noncommutative Jordan algebra, we can say more about the induced quadratic structure.

THEOREM 6. If $\mathfrak{A}$ is a noncommutative Jordan algebra then the quadratic algebra $\mathfrak{U}^{+}$with operations $x^{2}=x x$ and $U_{x} y=\left\{L_{x} V_{x}-L_{x^{2}}\right\} y=\left\{R_{x} V_{x}-R_{x^{2}}\right\} y$ is a quadratic Jordan algebra.

Proof. Firstly, it suffices to assume $\mathfrak{A}$ is unital. For any noncommutative Jordan algebra $\mathfrak{A}$ can be imbedded in a unital one $\mathfrak{X}^{\prime}$, and if $\mathfrak{U}^{\prime+}$ is quadratic Jordan then so is its subalgebra $\mathfrak{A}^{+}$.

Secondly, it suffices to prove such a unital $\mathfrak{A}^{+}$satisfies the axioms UCJ $1-5$, for the same holds for any extension $\left(\mathfrak{U}^{+}\right)_{\Omega}=\left(\mathfrak{U}_{\Omega}\right)^{+}$since $\mathfrak{A}_{\Omega}$ is again a unital noncommutative Jordan algebra.

We have already verified UCJ 1 and 2 in (5.5) and (5.4). In a noncommutative Jordan algebra the formula (5.1) and the commutativity of the multiplication operators $L_{x}, R_{x}, L_{x^{2}}, R_{x^{2}}$ gives UCJ 3,

(5.6) $U_{x} V_{x}=V_{x} U_{x}$.

There remains only to verify UCJ 4 and 5

(5.7) $U_{x^{2}}=U_{x}^{2}$

(5.8) $U_{x^{3}}=U_{x}^{3}$.

These verifications will take some effort. We introduce an associator operator

(5.9) $A_{x, y}(z)=[x, y, z]$ or $A_{x, y}=L_{x y}-L_{x} L_{y}$.

Note that since $x^{n}$ and $x^{m}$ operator-commute we have

(5.10) $A_{x^{n}, x^{m}}=A_{x^{m}, x^{n}}$.

In terms of the $A$ operator the linearized Jordan identity $\left[x^{2}, y, z\right]+[x \circ z, y, x]=0$ becomes $A_{x^{2}, y}(z)+A_{x_{0} z, y}(x)=0$. Now flexibility $A_{x, y}(x)=0$ implies $A_{x, y}(z)+$ $A_{z, y}(x)=0$, so we can interpret the previous identity as an operator identity

(5.11) $A_{x^{2}, y}=A_{x, y} V_{x}$.

From this we get an expression for $L_{x^{4}}: L_{x^{4}}=L_{x^{2}} L_{x^{2}}+A_{x^{2}, x^{2}}=L_{x^{2}}^{2}+A_{x, x^{2}} V_{x}=$ $L_{x^{2}}^{2}+A_{x^{2}, x} V_{x}=L_{x^{2}}^{2}+A_{x, x} V_{x}^{2}$ by (5.1), (5.11), (5.10), so

(5.12) $L_{x^{4}}=L_{x^{2}}^{2}+A_{x, x} V_{x}^{2}$.

Now (5.7) is easy: $U_{x^{2}}=L_{x^{2}} V_{x^{2}}-L_{x^{4}}\left(\right.$ by (5.1)) $=L_{x^{2}}\left\{V_{x}^{2}-2 U_{x}\right\}-\left\{L_{x^{2}}^{2}+A_{x, x} V_{x}^{2}\right\}$ (by (5.4), (5.12)) $=V_{x}^{2}\left\{L_{x^{2}}-A_{x, x}\right\}-L_{x^{2}}\left\{L_{x^{2}}+U_{x}\right\}-L_{x^{2}} U_{x}=V_{x}^{2}\left\{L_{x}^{2}\right\}-L_{x^{2}}\left\{L_{x} V_{x}\right\}$ $-U_{x} L_{x^{2}}$ (by (5.1), (5.9)) $=U_{x} L_{x} V_{x}-U_{x} L_{x^{2}}\left(\right.$ by (5.1)) $=U_{x} U_{x}$.

For (5.8) we need an expression for $L_{x^{3}}$. We have $L_{x^{3}}=L_{x^{2}} L_{x}+A_{x^{2}, x}$ $=\left\{L_{x} V_{x}-U_{x}\right\} L_{x}+A_{x, x} V_{x}=V_{x}\left\{L_{x}^{2}+A_{x, x}\right\}-U_{x} L_{x}=V_{x} L_{x}^{2}+V_{x}\left\{L_{x} V_{x}-U_{x}-L_{x}^{2}\right\}$ $-U_{x} L_{x}$ by (5.10), (5.1), (5.11),

(5.13) $L_{x^{3}}=L_{x} V_{x}^{2}-U_{x} V_{x}-U_{x} L_{x}$. 
Adding the dual relation for $R_{x^{3}}$ gives

(5.14) $V_{x^{3}}=V_{x}^{3}-3 V_{x} U_{x}$.

From this we compute $U_{x^{3}}=L_{x^{3}} V_{x^{3}}-L_{x^{6}}$ (by (5.1)) $=L_{x^{3}} V_{x^{3}}-\left\{L_{x^{2}} V_{x^{2}}^{2}-\right.$ $\left.U_{x^{2}} V_{x^{2}}-U_{x^{2}} L_{x^{2}}\right\}$ (by (5.13)) $=\left\{L_{x} V_{x}^{2}-U_{x} V_{x}-U_{x} L_{x}\right\}\left\{V_{x}^{3}-3 V_{x} U_{x}\right\}-\left\{L_{x} V_{x}-U_{x}\right\}$ $\cdot\left\{V_{x}^{2}-2 U_{x}\right\}^{2}+U_{x}^{2}\left\{V_{x}^{2}-2 U_{x}\right\}+U_{x}^{2}\left\{L_{x} V_{x}-U_{x}\right\}$ (by (5.13), (5.14), (5.1), (5.7)); when this is expanded out, using the commutativity of all the operators involved, it reduces to $U_{x}^{3}$, so $U_{x^{3}}=U_{x}^{3}$ and (5.8) is established.

COROLlaRY. If $\mathfrak{A}$ is a commutative Jordan algebra then $\mathfrak{A}^{+}$is a quadratic Jordan algebra with operations $x^{2}=x \cdot x, U_{x}=2 L_{x}^{2}-L_{x^{2}}$.

This result was first proved by I. G. Macdonald for the case when $\frac{1}{2} \in \Phi$. Both Theorem 6 and the corollary were proven for finite-dimensional algebras over a field (of arbitrary characteristic) in [5, p. 143].

If $\mathrm{FCJ}_{Z}(X)$ denotes the free commutative Jordan algebra on a set $X$ over the ring $Z$ of integers, $\mathrm{FCJ}_{Z}(X)^{+}$is a quadratic Jordan algebra, so we have a homomorphism

$$
\mathrm{FQJ}_{Z}(X) \rightarrow \mathrm{FCJ}_{Z}(X)^{+}
$$

from the free quadratic Jordan $Z$-algebra $\mathrm{FQJ}_{Z}(X)$ on $X$ sending $x \rightarrow x$ for $x \in X$. Professor N. Jacobson has raised the question as to whether this homomorphism is injective (it is not surjective); if this were the case then to verify that some identity holds in all quadratic Jordan algebras it would suffice to verify it in all commutative Jordan algebras. (Note that the corresponding map $\mathrm{FQJ}_{\Phi}(X) \rightarrow$ $\mathrm{FCJ}_{\Phi}(X)^{+}$is not injective if $\Phi$ has characteristic 2 , since in that case $V_{x}=2 L_{x}$ would be zero in $\mathrm{FCJ}_{\Phi}(X)^{+}$but $V_{x}$ is not zero in all special $\Phi$-algebras and so certainly not in $\mathrm{FQJ}_{\Phi}(X)$. Thus the bottom row of the diagram

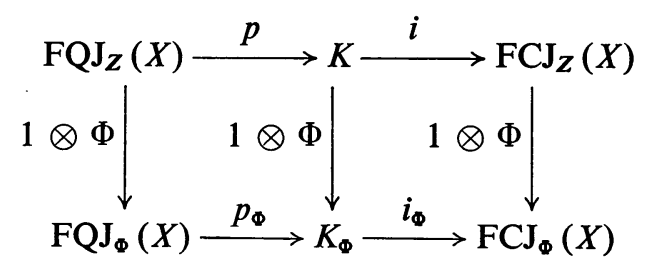

( $K$ the image of $\mathrm{FQJ}_{Z}(X)$ ) is not injective, but the top row could still be injective; of course, if it were then $p$ would be bijective, hence so would $p_{\Phi}$, but because $K$ is a proper subspace of $\mathrm{FCJ}_{Z}(X)$ the mapping $i_{\Phi}$ need not be an injection.)

\section{REFERENCES}

1. I. N. Herstein and E. Kleinfeld, Lie mappings in characteristic 2, Pacific J. Math. 10 (1960), 843-852. MR 22 \#5646.

2. M. Koecher, Eine Charakterisierung der Jordan-Algebren, Math. Ann. 148 (1962), 244-256. MR 26 \#2475. 
3. K. McCrimmon, A general theory of Jordan rings, Proc. Nat. Acad. Sci. U. S. A. 56 (1966), 1072-1079. MR 34 \#2643.

4. K. McCrimmon and R. D. Schafer, On a class of noncommutative Jordan algebras, Proc. Nat. Acad. Sci. U. S. A. 56 (1966), 1-4. MR 34 \#5888.

5. H. Braun and M. Koecher, Jordan-Algebren, Die Grundlehren der math. Wissenschaften in Einzeldarstellungen mit besonderer Berücksichtigung der Anwendungsgebiete, Band 128, Springer-Verlag, Berlin and New York, 1966. MR 34 \#4310.

UNIVERSITY OF VIRGINIA,

Charlottesville, Virginia 22901 